\title{
Condições hídricas de lavoura de soja usando sensoriamento remoto terrestre
}

\author{
Lucimara Wolfarth Schirmbeck ${ }^{1}$, Denise Cybis Fontana ${ }^{2(*)}$, Genei Antonio Dalmago ${ }^{3}$, Juliano Schirmbeck ${ }^{4}$, Paulo Roberto Vargas ${ }^{5}$ \\ e José Maurício Cunha Fernandes ${ }^{3}$ \\ ${ }^{1}$ Universidade Federal do Rio Grande do Sul, Programa de Pós-Graduação em Sensoriamento Remoto. Av. Bento Gonçalves, 9500, Campus do Vale, CEP \\ 91501-970 Porto Alegre, RS. E-mail: lucimaraws@gmail.com \\ ${ }^{2}$ Universidade Federal do Rio Grande do Sul, Faculdade de Agronomia. Av. Bento Gonçalves, 7712, Agronomia, CEP 91540-000 Porto Alegre, RS. \\ E-mail: dfontana@ufrgs.br \\ ${ }^{3}$ Embrapa Trigo. Rodovia BR 285, km 294, CEP 99050-970 Passo Fundo, RS. E-mail: genei.dalmago@embrapa.br e mauricio.fernandes@embrapa.br. \\ ${ }^{4}$ Universidade do Vale do Taquari, Centro de Ciências Exatas e Tecnológicas. Av. Avelino Talini, 171, CEP $95914-014$ Lajeado, RS. \\ E-mail: schirmbeck.j@gmail.com \\ ${ }^{5}$ Responsável técnico pela Fazenda Capão Grande. Carazinho, RS. E-mail: paulovargas@annex.com.br \\ ${ }^{(*)}$ Autor para correspondência.
}

\section{INFORMAÇÕES}

\section{História do artigo:}

Recebido em 18 de setembro de 2019

Aceito em 4 de março de 2020

Termos para indexação:

TVDI

NDVI

temperatura de superfície

\section{RESUMO}

O objetivo deste trabalho foi verificar o desempenho do índice TVDI (TemperatureVegetation Dryness Index), obtido a partir de sensores espectrais de superfície, e compará-lo a dados de déficit hídrico determinado pelo balanço hídrico meteorológico, em lavoura de soja no noroeste do Estado do Rio Grande do Sul. o princípio de funcionamento do TVDI está ancorado na inclinação da reta de regressão linear entre o índice de vegetação e a temperatura de superfície, que representam o grau de deficiência da umidade do sistema solo/água/planta. Para o estudo foram utilizados sensores de superfície de índice de vegetação, no caso o NDVI (Normalized Difference Vegetation Index) e de temperatura radiométrica da superfície, conectados a dataloggers, registrando medidas a cada 15 minutos. 0 TVDI foi comparado frente a dados de déficit e excesso hídrico obtidos por balanço hídrico meteorológico diário. No período de maior déficit o TVDI apresentou os maiores valores, indicando a restrição hídrica, coerente com o armazenamento de água no solo. As temperaturas de superfície também foram altas neste período. 0 TVDI estimado a partir de sensores de superfície tem sensibilidade em representar a disponibilidade hídrica da cultura e permite acompanhar o desenvolvimento da soja durante a safra.

(c) 2020 SBAgro. Todos os direitos reservados.

\section{Introdução}

No Rio Grande do Sul (RS) a agricultura está presente em todas as regiões, destacando o Estado no cenário brasileiro como um grande produtor de grãos. Conforme le- vantamento da CONAB (2018), a soja é uma das culturas que ocupa maior área e apresenta a maior produção, sendo que o RS produziu mais de 16,968 milhões de toneladas no ano de 2018. O RS é o terceiro maior produtor brasileiro de soja, com área semeada de 5,692 milhões de hectares. Já no 
cenário estadual a soja está se expandindo em áreas antes ocupadas pelo arroz e em conjunto com o arroz, o milho e o trigo constituem as principais culturas agrícolas (Feix et al., 2017).

Para as culturas de primavera verão, a disponibilidade de água se constitui como o principal fator limitante para a produção de grãos no RS (Matzenauer et al., 2002; Sentelhas et al., 2015; Zanon et al., 2016). A importância econômica da produção agrícola, aliada a busca constante pelo aumento da eficiência da produção, impulsionam e justificam a busca por novas tecnologias. Estas, devem ser capazes de viabilizar o monitoramento das condições hídricas, em uma larga escala geográfica e com alto detalhamento, tanto espacial quanto temporal. o uso do sensoriamento remoto já é realidade neste contexto e vem ganhando cada vez mais espaço, especialmente através de índices derivados das imagens orbitais (Huete et al., 2002, Ponzoni \& Shimabukuro, 2009; Sandholt et al., 2002). Estes índices devem ser, portanto, capazes de caracterizar as variações ocorridas entre lavouras dentro das regiões produtoras e também de forma contínua ao longo do crescimento e desenvolvimento das plantas. Isto é possível a partir da detecção da energia proveniente da superfície pelos sensores remotos, em distintas faixas do espectro eletromagnético, e do conhecimento de como as plantas interagem com a radiação solar.

Dentre diversos índices disponíveis, pode-se estimar as condições hídricas da superfície com uso do TVDI (Temperature-Vegetation Dryness Index), proposto por Sandholt et al. (2002). Um dos fatores que tornam este índice atrativo é o fato de que está baseado apenas no uso de dados aportados por imagens, que são: o índice de vegetação IV e a temperatura de superfície $\left(\mathrm{T}_{\mathrm{s}}\right)$. O princípio de funcionamento do TVDI está ancorado na dispersão de formato, geralmente triangular, que ocorre entre o índice de vegetação e a temperatura de superfície, também conhecido na literatura como triângulo evaporativo. A partir desta dispersão pode-se extrair os limites considerados seco e úmido, dentre os quais estima-se o índice TVDI. A inclinação da reta de regressão linear entre o índice de vegetação e a temperatura de superfície representa o grau de deficiência de umidade, dada a relação negativa entre estes dois parâmetros, conforme diversos estudos realizados (Venturini et al., 2004; Wang et al., 2007; Holzman et al., 2014).

Apesar da difusão do seu uso é ainda necessário testar e entender, de forma mais aprofundada, a aplicação do TVDI para condições de climas subtropicais úmidos, visto que grande parte dos trabalhos realizados são em condições de clima mais seco (Sandholt et al 2002; Mallick et al., 2009). Também, trabalhos realizados a campo permitem obter informações detalhadas da superfície, de forma controlada e com maior grau de precisão, em diversos momentos do desenvolvimento dos cultivos. Aliado a isto, ao se utilizar sensores na superfície, que possuem tecnologia similar a utilizada nos sensores a bordo dos satélites (Balzarolo et al., 2011) a tendência é minimizar os erros das estimativas, possibilitando uma compreensão mais realística das incertezas inerentes ao índice proposto.

o objetivo do trabalho foi verificar o desempenho do índice TVDI, obtido a partir de sensores espectrais de superfície, e compará-lo a dados de déficit hídrico determinado pelo balanço hídrico meteorológico diário, em experimento On Farm de soja no noroeste do estado do Rio Grande do Sul.

\section{Material e Métodos}

A área de estudo está localizada na região noroeste do Estado do RS, no município de Carazinho (Figura 1), em região caracterizada pela intensa produção de grãos no RS. O clima local, segundo Alvares et al. (2013), que tomou como base a classificação de Köppen, é subtropical úmido, com verões quentes e chuvas com distribuição regular ao longo do ano. O solo da propriedade é um Latossolo Vermelho Aluminoférrico tipico (Streck et al., 2008).

A lavoura avaliada ocupou uma área de 27,4 hectares dentro da área maior de 553,7 ha da Granja Capão Grande, que cultiva cerca de 455 ha de soja no verão. O experimento desenvolvido em formato On Farm, foi realizado em parceria entre a equipe de pesquisa em agrometeorologia da Faculdade de Agronomia da UFRGS e da Embrapa Trigo de Passo Fundo. A coleta de dados seguiu o método da amostragem (Surveys) simples em transectos localizados na parte central da área de estudo, cujo ponto central da mesma tinha, pelo menos, 100 metros de raio, em relação à borda da lavoura. Essa estratégia foi adotada, devido à coleta de dados micrometeorológicos, os quais necessitam desta condição de contorno para evitar a advecção de energia do meio externo à lavoura. A lavoura foi semeada em 13/11/2017 e a emergência ocorreu em 21/11/2017, com a colheita realizada em 03/04/2018. Foi utilizada a cultivar DM 5958 RSF IPRO, semeada no espaçamento de 45 $\mathrm{cm}$ entre linhas e densidade de 24 plantas. $\mathrm{m}^{-2}$. A adubação de base seguiu a análise de solo e correspondeu a $300 \mathrm{~kg}$ $\mathrm{ha}^{-1}$ de NPK, da fórmula 4-28-08 na semeadura e aplicação $160 \mathrm{~kg} \mathrm{ha}^{-1}$ de cloreto de potássio a lanço no dia anterior à semeadura. $\mathrm{O}$ controle de plantas daninhas, de pragas e de doenças foi realizado quando necessário, utilizando produtos indicados para a cultura (Reunião..., 2016), e foi baseado em monitoramento constante realizado no campo. Semanalmente, durante o ciclo da cultura foi registrado o estádio fenológico das plantas (Fehr \& Caviness, 1977) e ao final do ciclo foi determinado o rendimento de grãos, em quatro repetições de $9 \mathrm{~m}^{2}$ cada uma. O rendimento de grãos foi corrigido para $13 \%$ de umidade e calculado para uma área de um hectare. A lavoura foi conduzida sem irri- 
Figura 1. Localização da área de estudo no estado do Rio Grande do Sul, no município de Carazinho. Imagem Landsat-8 OLI de 7 fevereiro de 2018. Órbita/Ponto: 222/80. Composição RGB 6,5,4.

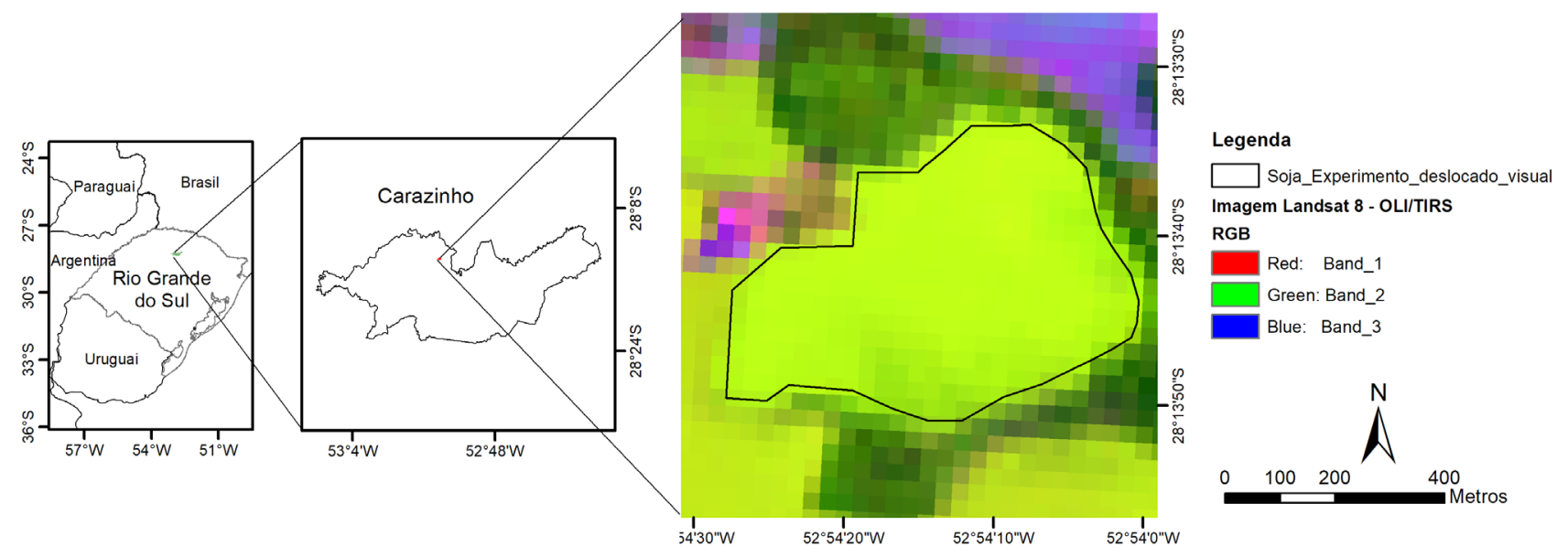

gação, sendo, portanto, a precipitação pluvial a única fonte de água para as plantas.

Os dados meteorológicos foram coletados através de estação meteorológica instalada na lavoura, com sensores a 1,5 m de altura do solo. Para a medição da temperatura e umidade relativa do ar foi utilizado um sensor conjugado, modelo HC2S3-L da Campbell Scientific, Inc. A radiação solar global incidente foi medida com um piranômetro modelo SP-110-L-10, da marca Apogee. 0 vento foi medido com um anemômetro sônico bidirecional, modelo WINDSONIC1-L34, marca GILL. A precipitação pluvial foi medida com o pluviômetro TB4-L da Campbell Scientific, Inc. Todos os sensores foram conectados a um datalogger modelo CR1000, da Campbell Scientific, Inc., e as leituras foram feitas a cada 30 segundos, com médias e/ou totalização, armazenadas a cada 15 minutos, conforme a variável.

Para a medição do NDVI foram instalados os sensores de índice de vegetação SRS - Decagon (NDVI - Normalized Difference Vegetation Index) e de temperatura radiométrica da superfície $\left(\mathrm{T}_{\mathrm{s}}\right)$, modelo SI 421, da Apogee (Figura 2). Os sensores de NDVI são de dois tipos. O sensor de NDVI hemisférico mede a radiação eletromagnética incidente, e o sensor de NDVI incidente mede a radiação refletida pela superfície. Ambos sensores de NDVI medem nos espectros do vermelho e infravermelho próximo e o sensor de $\mathrm{T}_{\mathrm{s}}$ mede a radiação emitida pela superfície no espectro do infravermelho termal. Os sensores são de monitoramento contínuo com registos a cada 15 minutos. Para registro dos dados foram utilizados dataloggers modelo CR 1000 da Campbell Scientific, Inc.. Estes sensores (NDVI e $\mathrm{T}_{\mathrm{s}}$ ) foram instalados em par, com ângulo de $90^{\circ}$ apontando para a mesma área, a uma altura de $1 \mathrm{~m}$ acima do topo do dossel da soja.

Para a estimativa do índice TVDI, no processo de parametrização, foram utilizados dados das imagens Landsat-8. A necessidade de uso das imagens se dá pelo fato de ser
Figura 2. Sensores utilizados nas medições durante o experimento para a cultura da soja na safra de 2017-2018. Da direita para esquerda: sensor de NDVI hemisférico, sensor de NDVI direcional SRS, marca Decagon e sensor de temperatura radiométrica $\left(\mathrm{T}_{\mathrm{S}}\right)$, modelo SI 421, marca Apogee. Carazinho - RS, 2018.

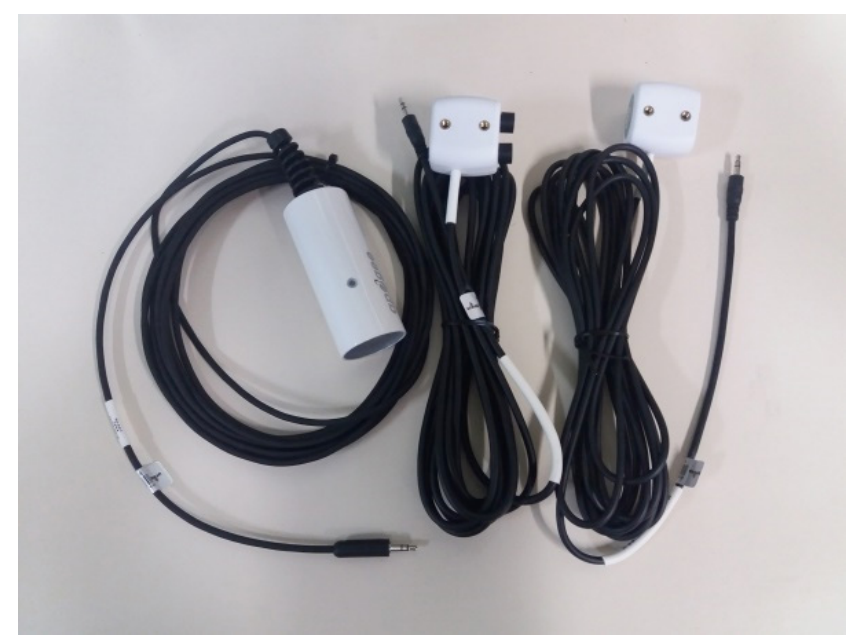

necessário que na área em estudo ocorram condições hídricas extremas, em termos de umidade do solo, para os diferentes valores de IV, de forma a propiciar uma correta determinação dos limites secos e úmidos do triângulo evaporativo (Roerink et al., 2000; Kustas et al., 2004), o que não é possível somente com medidas de escala local. Foi utilizada a técnica de parametrização por múltiplas imagens, de acordo com Schirmbeck et al. (2018a). Após parametrizado o TVDI foi estimado a partir dos dados de NDVI e $\mathrm{T}_{s}$ medidos em escala local aplicando a equação proposta por Sandholt et al. (2002):

$$
T V D I=\frac{\left(T_{S}-T_{S \min }\right)}{\left(a+b I V-T_{S \min }\right)}
$$

sendo: $\mathrm{T}_{\mathrm{S}}$ a temperatura radiativa do pixel (K); $\mathrm{T}_{\mathrm{smin}}$ a temperatura mínima de superfície (K) correspondente ao limi- 
te úmido na dispersão do triângulo evaporativo; IV o índice de vegetação no caso o NDVI; "a" e "b" os coeficientes linear e angular da reta que representam o limite seco do triângulo evaporativo.

A dispersão entre o NDVI e a $\mathrm{T}_{\mathrm{s}}$ geralmente apresenta uma forma triangular que depende da biomassa existente sobre o solo e do teor de umidade do solo (Figura 3) (Price, 1990; Sandholt et al., 2002; Garcia et al., 2014). No limite úmido do triângulo, onde se concentram as $\mathrm{T}_{\mathrm{s}}$ mínimas, o TVDI é zero e não há déficit hídrico. Já, no limite seco do triângulo, o TVDI é igual a 1 e ocorre déficit de umidade na superfície que está sendo imageada.

A partir dos dados de radiação solar global, precipitação pluvial, velocidade do vento, temperatura e umidade relativa do ar medidos na estação meteorológica instalada na lavoura foi estimada a evapotranspiração de referência pelo Método de Penman Monteith (FAO) (Allen et al., 2006) e após calculado o balanço hídrico meteorológico diário para obtenção dos dados de déficit e excesso hídrico. O balanço hídrico foi calculado usando a metodologia de Thornthwaite-Mather (1955), em escala diária (Pereira, 2005), considerando o Kc (entre 0,4 e 1,15) (Allen et al., 2006) e a CAD variável durante o ciclo em função do crescimento das plantas e as raízes ao longo do período (Dourado Neto et al., 1999). A soma térmica foi calculada conforme Rosa et al. (2009), com temperatura base de $10^{\circ} \mathrm{C}$ (Schneider et al., 1987).

Para a análise dos dados do TVDI foram escolhidos 25 dias ensolarados onde não houve interferência de nuvens ou sombras na hora da passagem do satélite Landsat. Como critério de seleção dos dias usou-se dados medidos de ra- diação solar global na estação meteorológica instalada no local.

\section{Resultados e Discussão}

Durante o período experimental, a precipitação pluvial ocorrida (Tabela 1) apresentou valores superiores à Normal Climatológica (1976-2005, Atlas Climático, 2011) em janeiro, fevereiro e março, meses considerados críticos quanto à condição hídrica para a cultura da soja cultivada na região. De novembro a maio a precipitação pluvial ocorrida foi de $770 \mathrm{~mm}$, e no período de maior desenvolvimento vegetativo (em janeiro) ocorreu $239 \mathrm{~mm}$ (Tabela 1). As condições ambientais ocorridas durante o ciclo da soja, proporcionaram rendimento de grãos de $4.629 \mathrm{~kg} \mathrm{ha}^{-1}$ (dados não apresentados).

No ciclo da cultura da soja, verificou-se variações no armazenamento de água no solo em função dos déficits e excessos hídricos ocorridos (Figura 4). No período inicial, em que a soja se encontrava nos estádios vegetativos (até início de janeiro), observou-se maior ocorrência de déficits hídricos diários com valores de até $4 \mathrm{~mm}$, o que acarretou em diminuições no armazenamento de água no solo em, praticamente, todo este período. A ocorrência de déficit hídrico no período vegetativo não necessariamente implica em redução no rendimento de grãos de soja. Durante o crescimento das plantas, danos causados por qualquer fenômeno adverso, inclusive déficit hídrico, poderão ser revertidos parcial ou totalmente se as condições do ambiente forem favoráveis no restante do ciclo (Bergamaschi \& Bergonci, 2017), que foi o que ocorreu na safra 2017-2018.
Figura 3. Dispersão entre o índice de vegetação NDVI e a temperatura de superfície (Ts), configurando o triângulo evaporativo. A reta em vermelho, onde o TVDI é 1 indica deficiência hídrica. A reta em azul é onde se localiza a temperatura de superfície mínima média (Tsmin) do período analisado e onde o TVDI é zero e não há deficiência hídrica. Dentro do triângulo é onde se localizam os diferentes tipos de cobertura no solo.

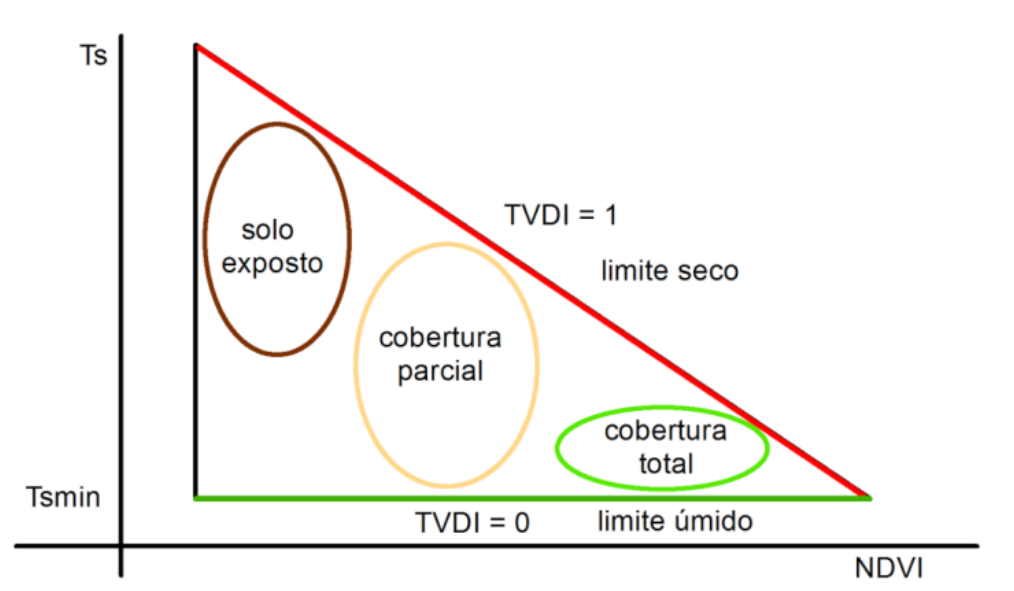

Tabela 1. Precipitação pluvial $(\mathrm{mm})$ ocorrida na área de estudo e a Normal Climatológica, no período da safra em valores mensais.

\begin{tabular}{|c|c|c|c|c|c|c|}
\hline \multirow{2}{*}{ Período } & \multicolumn{6}{|c|}{ Meses do ano } \\
\hline & Novembro & Dezembro & Janeiro & Fevereiro & Março & Abril \\
\hline Safra 2017-2018 & 102,0 & 70,1 & 238,5 & 154,4 & 201,4 & 3,3 \\
\hline Normal Climatológica & 155,2 & 166,2 & 158,7 & 146,5 & 135,3 & 133,5 \\
\hline
\end{tabular}


Figura 4. Componentes do balanço hídrico meteorológico diário durante o período experimental. EXC (excesso), DEF (déficit), CAD (Capacidade de água disponível no solo) e o ARMAZ (armazenamento de água no solo) para cultura da soja, na safra de $2017-2018$. Carazinho, RS, 2018.

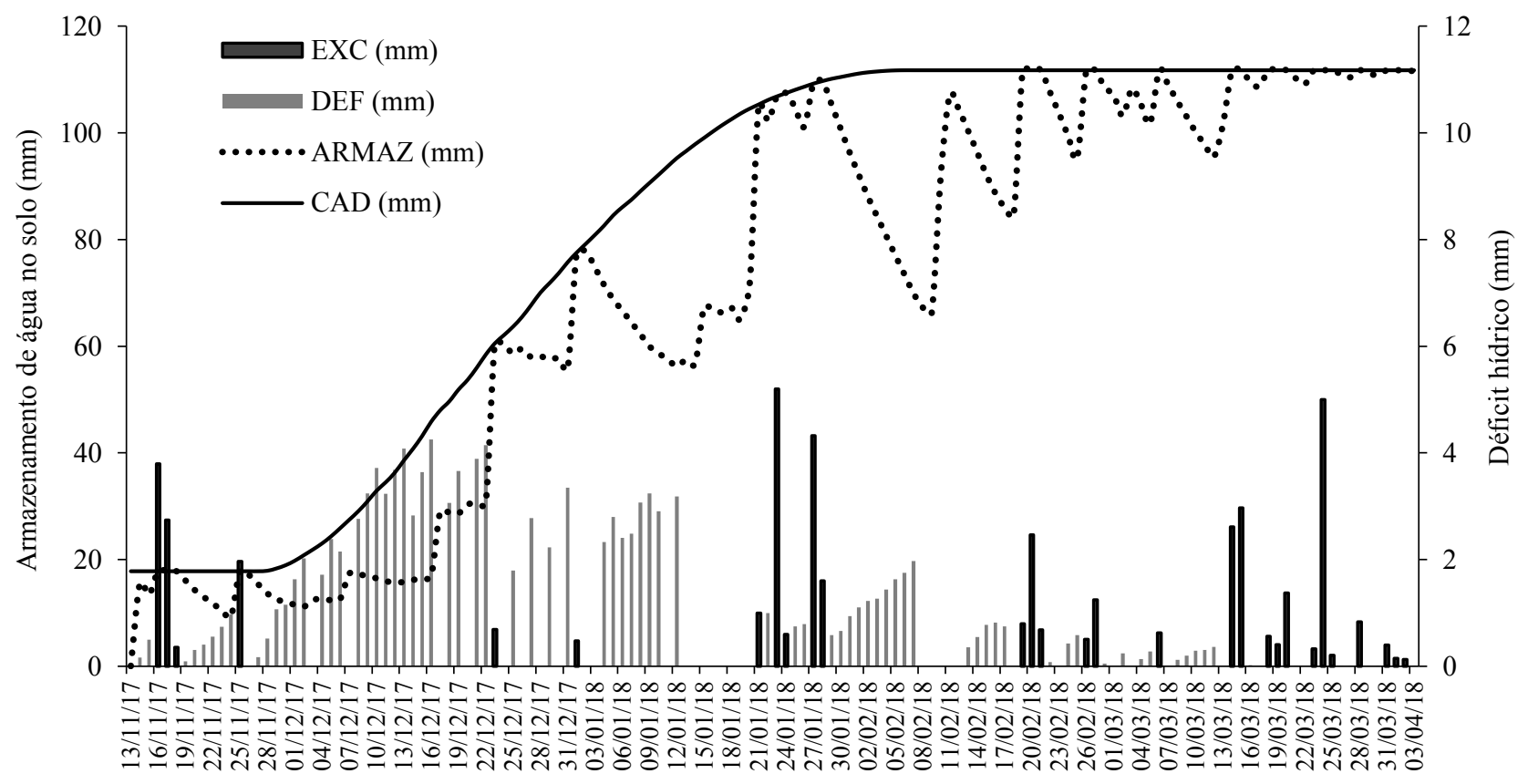

Data

Posteriormente, a condição hídrica se alterou e os déficits hídricos diminuíram em valores e frequência e se observou diversos períodos de excesso hídrico, de magnitudes superiores aos déficits, justamente no período reprodutivo. 0 período reprodutivo é o de maior consumo de água das plantas (Matzenauer et al., 2002; Mundstock \& Thomas, 2005) e define condições para obtenção de rendimentos de grãos elevados da soja.

A variação do índice de vegetação NDVI, ao longo do período experimental (Figura 5), caracterizou o crescimento das plantas de soja, visto que este índice é um dos estimadores remotos de biomassa verde que mais frequentemente tem sido utilizado (Ponzoni \& Shimabukuro, 2009). Observou-se no perfil de NDVI o padrão típico de lavouras anuais, com baixos valores de NDVI no início na implantação da cultura da soja, quando há presença de solo e restos de palha. Em seguida, ocorreu crescimento do índice, em decorrência do acúmulo de biomassa verde, até atingir um valor máximo, próximo a 0,9 , no florescimento. De acordo com Fontana et al. (2015), em geral o valor máximo ocorre no mês de fevereiro, quando a soja atinge o máximo índice de área foliar. Após o pico máximo o NDVI se manteve constante, praticamente, até o final do ciclo. Esta estabilidade nos valores do NDVI, mesmo com os incrementos de biomassa, denomina-se de saturação do índice e ocorreu em valores de NDVI próximo a 0,9 quando a vegetação atingiu o pleno desenvolvimento vegetativo nos estádios de R1 a R5.

A variabilidade dos dados da Ts, que é apresentado na
Figura 6, mostrou-se coerente com o esperado. No início da safra foram observadas as maiores Ts do solo, o que pode ser atribuído a duas causas principais. Primeiro, a maior disponibilidade de radiação solar global (próximo ao solstício de verão) e segundo a menor disponibilidade hídrica no solo, o que faz com que uma maior porção do saldo de radiação seja utilizada para o aquecimento do ar e do solo (Schirmbeck et al., 2018b). Portanto, a baixa cobertura do solo pela vegetação neste período inicial, propicia uma intensificação do aquecimento da superfície. Com o crescimento da vegetação nos períodos que seguem, esta passa a controlar, em parte, os fluxos de energia e a temperatura da superfície, através do processo de evapotranspiração, que se intensifica a partir do momento em que se verifica uma maior disponibilidade de água no solo (Goward et al., 2002; Liang et al., 2014).

A relação entre o déficit hídrico ocorrido e o índice TVDI ao longo da safra, pode ser avaliada na Figura 7. Observa-se que no início da safra, em dezembro, foi quando ocorreram os maiores déficits hídricos. Neste período também o índice TVDI apresentou os valores mais altos, indicando a restrição hídrica. Mais próximo ao final do ciclo foi quando ocorreram os menores valores de déficit hídrico, coincidindo com a ocorrência dos mais baixos valores de TVDI. Os dados evidenciaram, portanto, uma relação que tende a ser diretamente proporcional, em que aumentos e diminuições no déficit hídrico, resultaram em aumentos e diminuições proporcionais no TVDI. Esta foi, entretanto, apenas uma tendência geral, visto que neste período 
Figura 5. Perfil temporal do índice de vegetação NDVI (em verde), de 26 de dezembro de 2017 a 12 de março de 2018, para cultura da soja na safra de 2017-2018, com identificação dos estádios fenológicos do subperíodo vegetativo (V11 e V16) e reprodutivos (R1, R2, R3, R4, R5 e R6). Carazinho, RS, 2018.

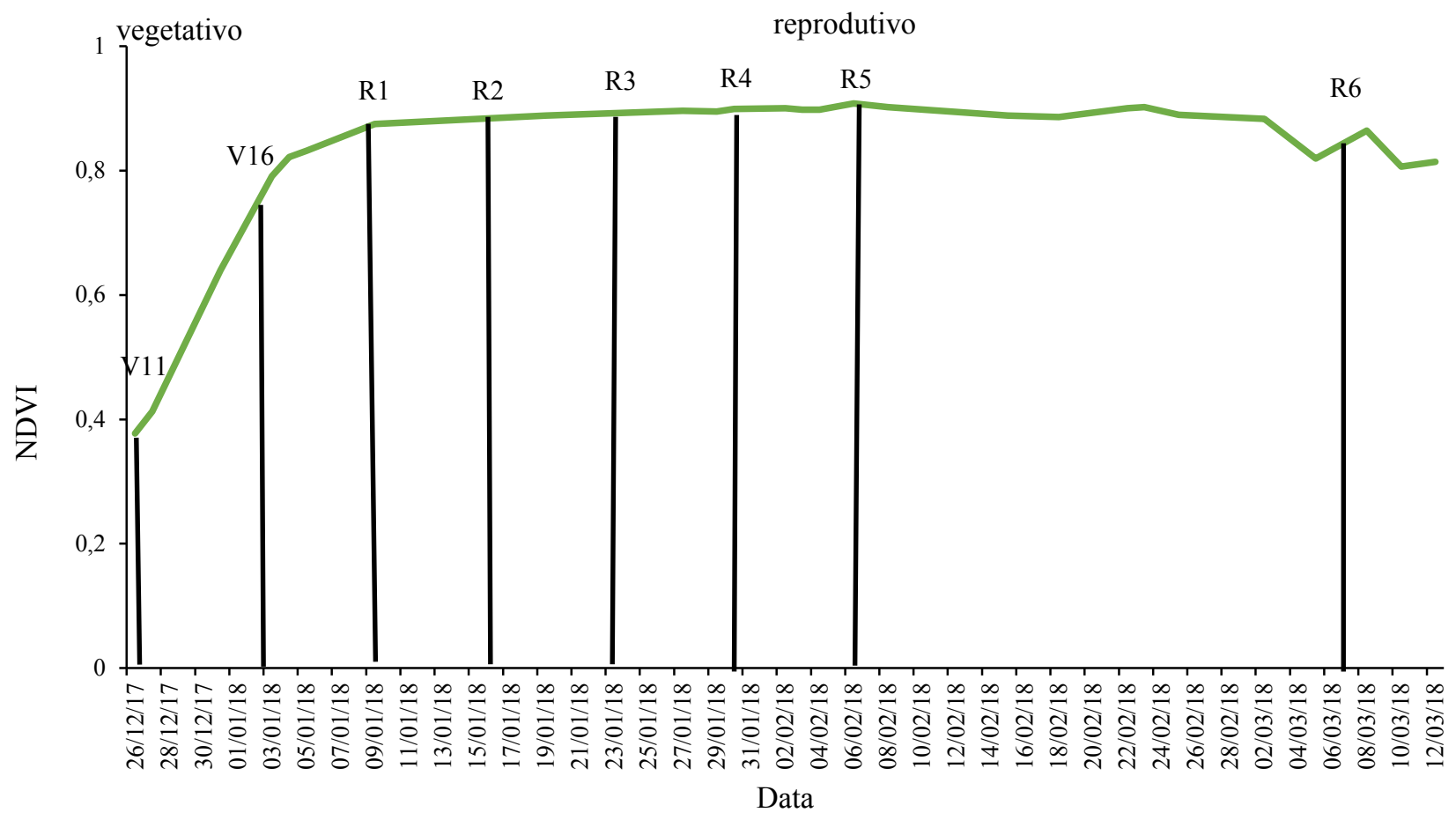

Figura 6. Perfil temporal da temperatura de superfície para cultura da soja de 26 de dezembro de 2017 a 12 de março de 2018 na safra de 2017-2018. Carazinho, RS, 2018.

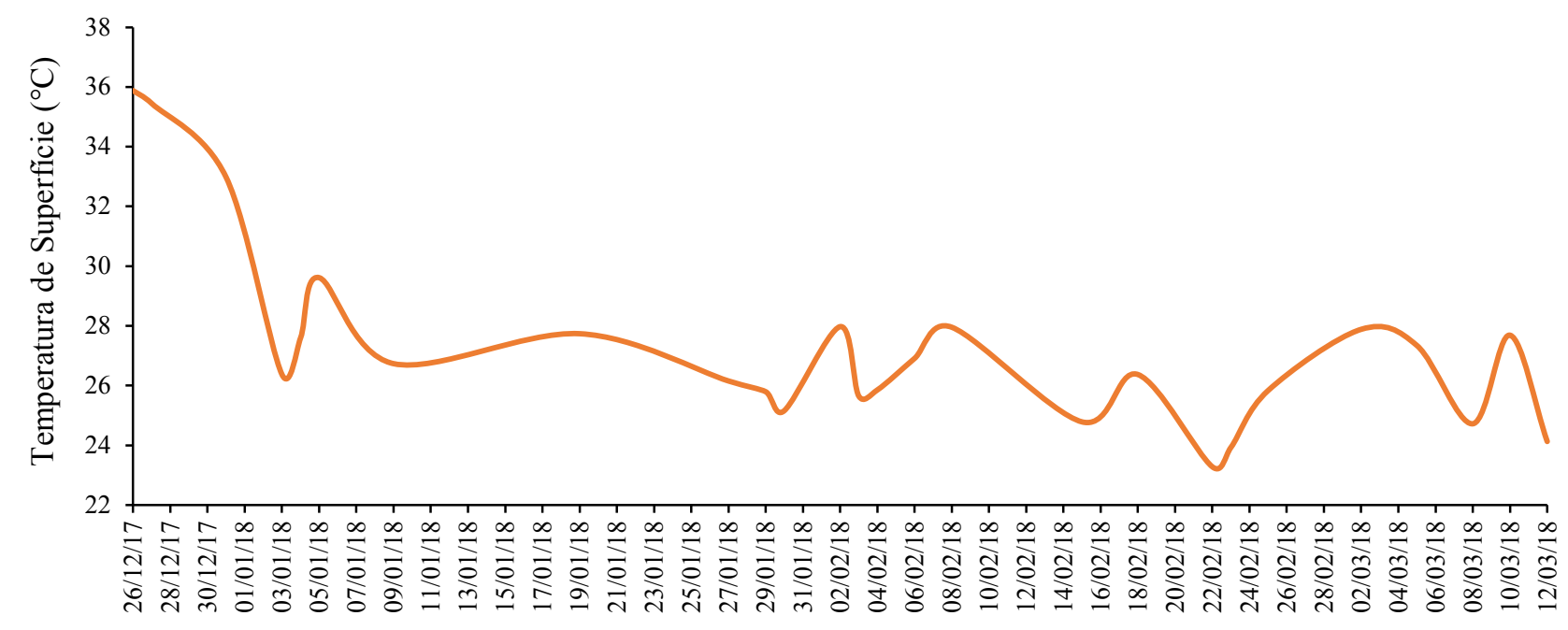

Data

final também foram observados valores altos de TVDI, principalmente nos dias 109, 112, e 117 após a semeadura da soja, quando se observou menor nebulosidade e maior temperatura de superfície em relação aos demais dias do período, além de que a soja estava entrando em maturação fisiológica. Holzman et al. (2014) constataram que em áreas com clima úmido, a variabilidade nos parâmetros do TVDI é mais evidente em anos onde o regime de precipitação pluvial apresenta-se abaixo do normal. Este não foi o caso da safra 2017-2018 analisada, mas em estudos futuros isto deverá ser avaliado.

\section{Conclusão}

O TVDI estimado a partir de sensores de superfície mostra sensibilidade em representar a disponibilidade hídrica no sistema solo/água/planta e permite acompanhar a condição hídrica durante o desenvolvimento da safra.

No período de maior déficit hídrico, os valores de TVDI são maiores, indicando a restrição hídrica à cultura, o que também pode ser verificado avaliando o armazenamento de água no solo, assim como através dos dados de temperaturas da superfície. 
Figura 7. Transcurso temporal do déficit hídrico do solo, obtido pelo cálculo do balanço hídrico diário e transcurso do índice TVDI (os marcadores em formato circular no interior da figura representam o número de dias após a semeadura) para cultura da soja na safra de 2017-2018. Carazinho, RS, 2018.

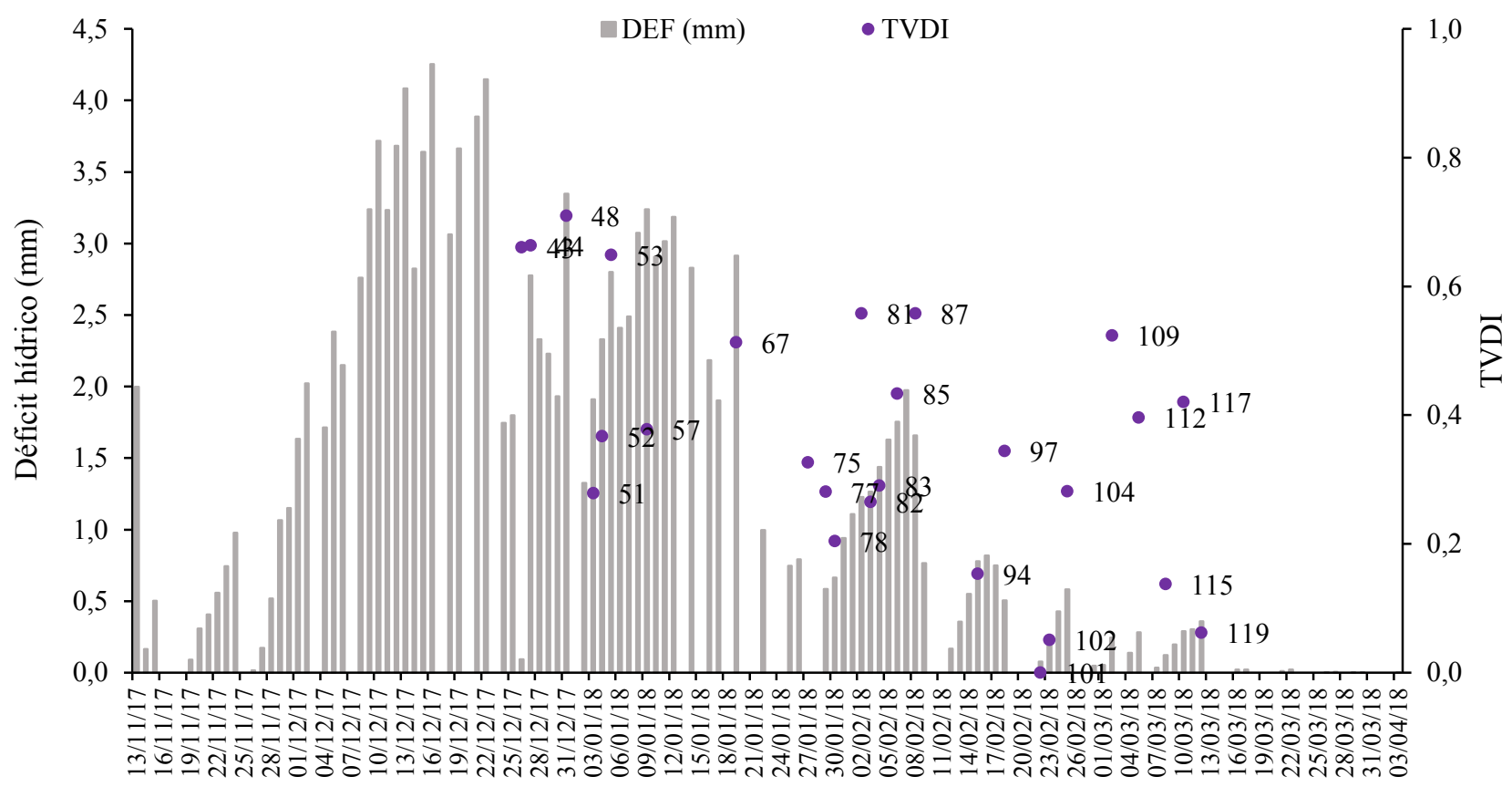

Data

\section{Agradecimentos}

O presente trabalho foi realizado com apoio da Coordenação de Aperfeiçoamento de Pessoal de Nível Superior - Brasil (CAPES) - Código de Financiamento 001, do Conselho Nacional de Desenvolvimento Científico e TecnológiCo (CNPq) e pela Embrapa Trigo, por meio do projeto SEG 02.15.07.003.00.00. A equipe agradece também aos funcionários da Embrapa Trigo, Elisson S. S. Pauletti e Cristian M. Plentz, que auxiliaram na condução do experimento e coletas dos dados e a Granja Campão Grande pela parceria no trabalho.

\section{Referências}

ALLEN, R. G., PEREIRA, L. S., RAES, D., SMITH, M. Evapotranspiración del cultivo: guías para la determinación de los requerimientos de agua de los cultivos. Roma: FAO. 2006, 323p. (FAO: Irrigation and Drainage Paper, 56).

ALVARES, C.A.; STAPE, J.L.; SENTELHAS, P.C.; GONÇALVES, J.L.M.; SPAROVEK, G. Köppen's climate classification map for Brazil. Meteorologische Zeitschrift 22:711-728. 2013.

BALZAROLO, M.; ANDERSON, K.; NICHOL, C.; ROSSINI, M.; VESCOVO, L.; ARRIGA, N.; CALVET, J.-C.; CARRARA, A.; CERASOLI SALVATORI, S.; COGLIATI, S.; et al. Ground-based optical measurements at European flux sites: A review of methods, instruments and current controversies. Sensors, v. 11, p. 7954-7981. 2011.

BERGAMASCHI, H., BERGONCI, J. I. As Plantas e o Clima - Princípios e Aplicações. Guaiba: Agrolivros, 2017. 352p.

Companhia Nacional de Abastecimento CONAB. Acompanhamento safra brasileira de grãos, v. 5 - Safra 2017/18, n 8 - Oitavo levantamento, maio 2018 .
DOURADO NETO, D.; GARCÍA, A. G.; FANCELLI, A. L.; FRIZZONE, J. A.; REICHARDT, K. Balance hídrico cíclico y secuencial: estimación de almacenamiento de agua em el suelo. Scientia Agricola, v. 56, n. 3, p. 537-546, 1999.

FEIX, R. D., LEUSIN JÚNIOR, S., AGRANONIK, C. Painel do agronegócio no Rio Grande do Sul - 2017. Porto Alegre: Fundação de Economia e Estatística - FEE, 2017.

FEHR, W. R.; CAVINESS, C. E. Stages of soybean development. Ames: Iowa State University of Science and Technology, 1977. 15p. (Special Report, 80).

FONTANA, D. C.; PINTO, D. G.; JUNGES, A. H., BREMM, C. Inferências sobre o calendário agrícola a partir de perfis temporais de NDVI/MODIS. Bragantia, Campinas, v. 74, n. 3, p. 350-358, 2015.

GARCIA, M., FERNÁNDEZ N., VILLAGARCÍA, L., DOMINGO, F., PUIGDEFÁBREGAS, J., SANDHOLT, I. Accuracy of the TemperatureVegetation Dryness Index using MODIS under water-limited vs. energy-limited evapotranspiration conditions. Remote Sensing of Environment, New York, v. 149, p. 100-117, 2014.

GOWARD, S. N., XUE, Y., CZAJKOWSKI, K. P., Evaluating land surface moisture conditions from the remotely sensed temperature/vegetation index measurements. An exploration with the simplified simple biosphere model. Remote Sensing of Environment, New York, v. 79, n. 3-3, p. 225-24, 2002.

HOLZMAN, M.E.; RIVAS, R.E.; PICCOLO, M.C. Estimating soil moisture and the relationship with crop yield using surface temperature and vegetation index. International Journal of Applied Earth Observation and Geoinformation, v. 28, p. 181-192. 2014.

HUETE, A., DIDAN, K., MIURA, T., RODRIGUEZ, E. P., GAO, X., FERREIRA, L. G. Overview of the radiometric and biophysical performance of the MODIS vegetation indices. Remote Sensing of Environment, v. 83, p.195-213. 2002.

KUSTAS, W.P., LI, F., JACKSON, T.J., PRUEGER, J.H., MACPHERSON, J.L., WOLDE, M., Effects of remote sensing pixel resolution on model edenergy flux variability of croplands in Iowa. Remote Sensing of Environment, New York, v. 92, n. 4, p. 535-547, 2004. 
LIANG, L., ZHAO, S., QIN, Z., HE, K., CHEN, C., LUO, Y., ZHOU, X., Drought Change Trend Using MODIS TVDI and Its Relationship with Climate Factors in China from 2001 to 2010. Journal of Integrative Agriculture, Beijing, v.13, n. 7, p. 1501-1508, 2014.

MALLICK, K.; BHATTACHARYA, B.K.; PATEL, N.K. Estimating volumetric surface moisture content for cropped soils using a soil wetness index based on surface temperature and NDVI. Agricultural and Forest Meteorology, v. 149, p. 1327-1342. 2009.

MATZENAUER, R.; BERGAMASCHI, H.; BERLATO, M.A.; MALUF, J.R.T.; BARNI, N.A.; BUENO, A.C.; DIDONÉ, I.A.; ANJOS, C.S.; MACHADO, F.A.; SAMPAIO, M.R. Boletim FEPAGRO, Consumo de água e disponibilidade hídrica para milho e soja no Rio Grande do Sul, n. 10. 2002.

MATZENAUER, R.; RADIN, B.; ALMEIDA, I.R. (Ed.). Atlas Climático: Rio Grande do Sul. Porto Alegre: Secretaria da Agricultura Pecuária e Agronegócio; Fundação Estadual de Pesquisa Agropecuária (FEPAGRO), 2011.

MUNDSTOCK, C., \& THOMAS, A. L. Soja: Fatores que afetam o crescimento e o rendimento de grãos. Departamento de Plantas de Lavoura da Universidade Federal do Rio Grande do Sul, Porto Alegre: Evangraf, 2005. 31p.

PEREIRA, A. R. Simplicando o balanço hídrico de Thornthwaite-Mather. Bragantia, v. 64, n. 2, p. 311-313, 2005.

PONZONI, F.J.; SHIMABUKURO, Y.E. Sensoriamento remoto no estudo da vegetação. São José dos Campos: Parêntese, 2009. 127 p.

PRICE, J. C. Using spatial context in satellite data to infer regional scale evapotranspiration. IEEE Transactions on Geoscience and Remote Sensing, New York, v. 28, n. 5, p. 940-948, 1990.

Reunião de Pesquisa de Soja da Região Sul. Indicações técnicas para a cultura da soja no Rio Grande do Sul e em Santa Catarina, safras 2016/2017 e 2017/2018. [Organizadores] José Roberto Salvadori ... et al. - Passo Fundo: Ed. Universidade de Passo Fundo, 2016. 128p.

ROERINK, G. J., Su, B., \& MENENTI, M. S-SEBI: A simple remote sensing algorithm to estimate the surface energy balance. Physics and Chemistry of the Earth, Part B, Hydrology, Oceans and Atmosphere, Oxford, v. 25, n. 2, p. 147-157, 2000.
ROSA, H. T.; WALTER, L. C.; STRECK, N. A.; ALBERTO, C. M. Thermal time methods and sowing dates in phyllochron determination in wheat cultivars. Pesquisa Agropecuária Brasileira, v. 44, n. 11, p. 1374-1382, 2009.

SANDHOLT, RASMUSEN, K., ANDERSEN, J. A simple interpretation of the surface temperature/vegetation index space for assessment of surface moisture status. Remote Sensing of Enviromenmt, 79, 213-224. 2002.

SENTELHAS, P.C., BATTISTI, R., CÂMARA, G.M.S., FARIAS, J.R.B., HAMPF, A.C., NENDEL, C., The soybean yield gap in Brazil: magnitude, causes and possible solutions for sustainable production. Journal of Agricultural Science 153, 1394-1411. 2015.

SCHIRMBECK, L. W., FONTANA, D. C., SCHIRMBECK, J. Two approaches to calculate the TVDI in the humid subtropical climate of southern Brazil. Scientia Agricola v. 75, n. 2, p. 111-120. 2018a.

SCHIRMBECK, J. FONTANA, D. C., ROBERTI, D. R. Evaluation of OSEB and SEBAL models for energy balance of a crop area in a humid subtropical climate. Bragantia, vol.77, n.4, pp.609-621. 2018 b.

SCHNEIDER, F. M.; BURIOL, G. A.; HELDWEIN, A. B.; MANFRON, P. A.; SACCOL, A. V.; ESTEFANEL, V. Temperatura base e soma térmica do subperíodo semeadura-emergência para algumas cultivares de soja (Glycine max (L.) Merrill). Revista do Centro de Ciências Rurais, v. 11, n. 4, p.299-308, 1987.

STRECK, E.V.; KÄMPF, N.; DALMOLIN, R. S. D.; KLAMT, E.; NASCIMENTO, P. C.; SCHNEIDER, P.; GIASSON, E.; PINTO L. F. S. Solos do Rio Grande do Sul. 2 ed. Porto Alegre: Emater/RS, 2008. 222 p.

THORNTHWAITE, C. W., MATHER, J. R. The water balance. Publications in Climatology. New Jersey: Drexel Institute of Technology, 1955, 104p.

VENTURINI, V., BISHT, G., \& ISLAM, S. Comparison of evaporative fractions estimated from AVHRR and MODIS sensors over South Florida. Remote Sensing of Environment, New York, v. 93, n. 1-2, p. 77-86, 2004.

WANG, X.; XIE, H.; GUAN, H. Different responses of MODIS-derived NDVI to root-zone soil moisture in semi-arid and humid regions. Journal of Hydrology, v. 340, p. 12-24. 2007.

\author{
REFERENCIAÇÃO $\quad$ SCHIRMBECK, L. W.; FONTANA, D. C.; DALMAGO, G. A.; SCHIRMBECK, J.; VARGAS, P. R. V.; \\ FERNANDES, J. M. C. Condições hídricas de lavoura de soja usando sensoriamento remoto terrestre. \\ Agrometeoros, Passo Fundo, v.27, n.1, p.173-181, set 2019.
}

Declaração: os trabalhos estão sendo publicados nesse número de AGROMETEOROS (v.27, n.1, set 2019) conforme foram aceitos pelo XXI Congresso Brasileiro de Agrometeorologia, realizado de 12 a 16 de agosto de 2019, em Catalão, Goiás, sem revisão editorial adicional da revista. 


\title{
Water conditions of soybean crop using ground remote sensing
}

\author{
Lucimara Wolfarth Schirmbeck ${ }^{1}$, Denise Cybis Fontana ${ }^{2(*)}$, Genei Antonio Dalmago ${ }^{3}$, Juliano Schirmbeck ${ }^{4}$ Paulo Roberto Vargas $^{5}$ \\ and José Maurício Cunha Fernandes ${ }^{3}$ \\ ${ }^{1}$ Universidade Federal do Rio Grande do Sul, Programa de Pós-Graduação em Sensoriamento Remoto. Av. Bento Gonçalves, 9500, Campus do Vale, CEP \\ 91501-970 Porto Alegre, RS, Brazil. E-mail: lucimaraws@gmail.com \\ ${ }^{2}$ Universidade Federal do Rio Grande do Sul, Faculdade de Agronomia. Av. Bento Gonçalves, 7712, Agronomia, CEP $91540-000$ Porto Alegre, RS, Brazil. \\ E-mail: dfontana@ufrgs.br \\ ${ }^{3}$ Embrapa Trigo. Rodovia BR 285, km 294, CEP 99050-970 Passo Fundo, RS, Brazil. E-mail: genei.dalmago@embrapa.br and \\ mauricio.fernandes@embrapa.br \\ ${ }^{4}$ Universidade do Vale do Taquari, Centro de Ciências Exatas e Tecnológicas. Av. Avelino Talini, 171, CEP 95914-014 Lajeado, RS, Brazil. \\ E-mail: schirmbeck.j@gmail.com \\ ${ }^{5}$ Responsável técnico pela Fazenda Capão Grande. Carazinho, RS, Brazil. E-mail: paulovargas@annex.com.br \\ ${ }^{(*)}$ Corresponding author.
}

\section{ARTICLE INFO}

Article history:

Received 18 September 2019

Accepted 4 March 2020

\section{Index terms:}

TVDI

NDVI

surface temperature

\section{ABSTRACT}

The objective of this work was to verify the performance of the TemperatureVegetation Dryness Index (TVDI), obtained from surface spectral sensors, and to compare it with water deficit data determined by the meteorological water balance, in soybean crops in the northwest of Brazil. State of Rio Grande do Sul. The principle of operation of TVDI is anchored in the slope of the linear regression line between vegetation index and surface temperature, which represent the degree of soil / water / surface system moisture deficiency. For the study we used vegetation index surface sensors, in this case the NDVI (Normalized Difference Vegetation Index) and radiometric surface temperature sensors, connected to dataloggers, recording measurements every 15 minutes. TVDI was compared against water deficit and excess data obtained by daily meteorological water balance. In the period of greatest deficit, TVDI showed the highest values, indicating water restriction, consistent with soil water storage. Surface temperatures were also high during this period. Estimated TVDI from surface sensors is sensitive to represent crop water availability and allows to monitor soybean development during the crop.

(C) 2020 SBAgro. All rights reserved.

\footnotetext{
CITATION

SCHIRMBECK, L. W.; FONTANA, D. C.; DALMAGO, G. A.; SCHIRMBECK, J.; VARGAS, P. R. V.;

FERNANDES, J. M. C. Condições hídricas de lavoura de soja usando sensoriamento remoto terrestre. Agrometeoros, Passo Fundo, v.27, n.1, p.173-181, set 2019.
}

Disclaimer: papers are published in this issue of AGROMETEOROS (v. 27, n.1, set 2019) as accepted by the XXI Congresso Brasileiro de Agrometeorologia, held August 12-16, 2019 in Catalão, Goiás State , Brazil, without further revision by editorial board.

Agrometeoros, Passo Fundo, v.27, n.1, p.173-181, set 2019. 18 